\title{
ENGEVISTA
}

Página da revista: http://www.uff.br/engevista/seer/

\section{Análise dos Impactos da Substituição de Lâmpadas Fluorescentes por Lâmpadas LEDs em Navios Militares}

\author{
Márcio Zamboti Fortes ${ }^{1}$ \\ Larissa de Cássia dos Santos Gouveia ${ }^{2}$ \\ Thiago Almeida Rodrigues ${ }^{3}$ \\ Fernando José Oliveira ${ }^{4}$ \\ Lorenna Baptista Oliveira ${ }^{5}$
}

Resumo: A busca pela eficiência e um melhor gerenciamento da energia elétrica em embarcações têm se tornado cada vez mais constante. Uma maneira simples e eficaz de contribuir para a melhoria da eficiência energética nos navios é a substituição de lâmpadas fluorescentes por lâmpadas LEDs (Light Emitting Diode). Além do aumento da eficiência, os LEDs trazem significantes benefícios para a rede elétrica do navio, como por exemplo, a melhoria do fator de potência da instalação e da qualidade da energia, além de redução da manutenção devido ao seu maior tempo de vida útil. O presente trabalho apresenta um estudo de caso a ser implementado em um navio de pequeno porte da Marinha do Brasil, para a substituição de lâmpadas fluorescentes por lâmpadas LEDs. Serão analisados, de forma detalhada, os benefícios que a utilização do LED traz para sob vários aspectos para o navio. Além das vantagens técnicas associadas à substituição, será avaliado também o aspecto econômico, considerando o investimento inicial, economia de combustível da fonte térmica geradora de energia e o tempo de vida útil

Palavras-chave: LED; eficiência energética; navios militares; qualidade de energia.

\footnotetext{
${ }^{1}$ UFF - Universidade Federal Fluminense

2 UFF - Universidade Federal Fluminense

${ }^{3}$ UFF - Universidade Federal Fluminense

${ }^{4}$ UFF - Universidade Federal Fluminense

${ }^{5}$ UFF - Universidade Federal Fluminense
} 
ISSN: $1415-7314$

ISSN online: $2317-6717$

Abstract: The searches for efficiency and better management of the electric power in vessels have become increasingly constant. A simple and effective way to improve the energy efficiency in ships is the replacement of fluorescent lamps by LED (Light Emitting Diode) lamps. Besides this increased efficiency, LEDs bring significant benefits to the ship's power grid, such as improving the power factor and energy quality of the ship, and reducing maintenance due to its longer useful life. The current work presents a case study to be implemented in a Brazilian Navy small ship, for the replacement of fluorescent lamps by LED lamps. The benefits that the LED will bring to the vessel will be analyzed in detail. In addition to technical advantages associated with substitution, the economic aspect will also be evaluated, considering the initial investment, fuel economy of the thermal power generating source and the useful life.

Keywords: LED; energy efficiency; military ships; energy quality. 


\section{Introdução}

A eficiência energética vem ganhando cada vez mais importância no segmento industrial, inclusive na indústria militar naval. As principais Marinhas do mundo, como por exemplo, a Marinha Americana, está cada vez mais empenhada em reduzir o consumo de combustível da frota, além de torná-la mais ecológica (McCoy, 2012; Morton e Truver, 2011). A busca pela redução no consumo de combustível nas embarcações justifica-se por diversos fatores, destacando como principal deles o fato de ser utilizado óleo diesel como principal fonte de energia, esse óleo é utilizado tanto para propulsão quanto para a geração de energia elétrica do navio, o que contribui sobremaneira com a emissão de poluentes na atmosfera (Marpol, 2018). De forma a reduzir tanto o custo operacional da embarcação como o consumo de combustível, são propostas nas literaturas diversas metodologias para melhorar a eficiência e o gerenciamento da energia nas embarcações (Boveri et al 2017; Brooks e Tribble, 2014; Sarris, 2011).

Uma forma simples e eficaz de melhorar a eficiência energética dos navios é a utilização da tecnologia LED (Light Emitting Diode) para iluminação, uma vez que esta possui eficiência luminosa muito superior à lâmpada fluorescente, sendo esta última ainda muito utilizada, especialmente na Marinha do Brasil (Brooks e Tribble, 2014).

Pela análise de demanda de um navio de guerra, a iluminação geral, desconsiderando iluminação de hangar, luzes de navegação e outros tipos de iluminação especial, corresponde a aproximadamente 5\% de toda "carga hotel" (que inclui sistemas auxiliares, ar condicionado, ventiladores e iluminação) (Cizec, 2009).

Além do significativo aumento na eficiência luminosa, a utilização de lâmpadas LEDs traz consigo vários benefícios em sua utilização. No que diz respeito à qualidade de energia, principalmente em relação à produção de correntes harmônicas, aspecto sensível em redes elétricas de navios devido às características peculiares de sistemas isolados, as lâmpadas LEDs também possuem um desempenho superior em relação às lâmpadas fluorescentes (Sola e Salichs, 2007; Bravo e Abed, 2013). Outro benefício significativo do LED é a redução significativa da manutenção, diminuição de sobressalentes e aumento da confiabilidade (Freymiller, 2009), que pode impactar na possibilidade de reduzir o pessoal de bordo, diminuindo assim o custo operacional do navio. Uma das principais dificuldades para se implementar a iluminação LED em navios da Marinha do Brasil é o custo inicial para adoção da tecnologia em toda a embarcação, o valor da lâmpada LED ainda é substancialmente superior a fluorescente, apesar do custo ser potencialmente diluído no decorrer da vida útil do navio. Um estudo realizado na Marinha dos Estados Unidos mostra, para as diversas classes de navios de sua frota, qual o tempo de retorno do investimento para a referida classe, sendo que alguns não trazem o retorno do investimento ao longo de sua vida útil (Cizec, 2009). Somado as questões objetivas que relacionam as vantagens e desvantagens da utilização de lâmpadas LEDs em navios de guerra da Marinha do Brasil, há 
ainda aspectos organizacionais subjetivos. Não é uma tarefa simples convencer organizações tradicionais que é importante adotar novas tecnologias (Dew et al., 2017).

Este artigo busca avaliar a implementação de iluminação LED em detrimento das lâmpadas fluorescentes, considerando apenas a iluminação geral, de um navio militar de pequeno porte, que será construído pela Marinha do Brasil.

\section{Metodologia}

Com o objetivo de comparar e validar o desempenho das tecnologias de lâmpadas LEDs e fluorescente, para aplicação no sistema de iluminação geral no projeto de uma embarcação militar da Marinha do Brasil, foram realizados os seguintes testes no laboratório de luminotécnica da Universidade Federal Fluminense (UFF), Lablux.

- Curva IES (Illumination Engineering Society);

- FluxoLuminoso (lm);

- EficiênciaLuminosa $(\mathrm{lm} / \mathrm{W})$;

- Temperatura de cor $(\mathrm{K})$;

- Índice de Reprodução de Cor - IRC;

- Potência Nominal;

- Fator de Potência e

- Distorção Harmônica Total (THD).

Para a realização dos testes, foram montadas em luminárias adequadas para uso naval duas lâmpadas de cada tecnologia a ser comparada. O primeiro teste foi realizado com duas lâmpadas PHILIPS fluorescentes tubulares de 20W cada, e o segundo com duas lâmpadas OSRAM Tuboled de 10W cada. Todos os testes foram realizados de acordo com as portarias do INMETRO recomendadas para tal (INMETRO, 2010; INMETRO, 2014; INMETRO, 2015). A tensão de alimentação utilizada na realização dos testes foi de $127 \mathrm{~V}$, compatível com a tensão do circuito de alimentação do sistema de iluminação da embarcação. Os seguintes equipamentos foram utilizados para a realização dos testes fotométricos, obtenção do espectro radiométrico e parâmetros elétricos:

- GO2000 - A Goniophotometer;

- HAAS-2000;

- Fonte de Tensão Pacific e

- Wattímetro Yokogawa WT-230.

A partir dos resultados obtidos em laboratório, será realizada uma análise geral sobre o ponto de vista técnico, analisando a eficiência energética de cada tecnologia proposta, e econômica, verificando os custos com a energia gasta para cada tipo de luminária além da 
quantidade de sobressalentes necessários durante todo o ciclo de operação do navio, de forma a verificar o tempo estimado de retorno do investimento para a adoção da tecnologia LED.

\section{Resultados}

As principais características das luminárias comparadas nos testes são apresentadas na tabela 1. O desempenho da luminária LED, conforme esperado, é muito superior em todos os aspectos comparados. Com relação à eficiência luminosa, principal objetivo da substituição do sistema e iluminação, a luminária fluorescente apresenta um valor de 46,62\% da eficiência luminosa da luminária LED.

Tabela 1: Comparação dos Dados Medidos

\begin{tabular}{|c|c|c|}
\hline & $\begin{array}{c}\text { Fluorescente } \\
(2 \times 20 \mathrm{~W})\end{array}$ & $\begin{array}{c}\text { LED } \\
(2 \times 10 \mathrm{~W})\end{array}$ \\
\hline Temperatura de Cor (K) & 4671 & 5146 \\
\hline $\begin{array}{c}\text { Indice Reprodução de Cor } \\
\text { (IRC) }\end{array}$ & $66,8 \%$ & $83,8 \%$ \\
\hline Eficiência Luminosa (lm/W) & 45,36 & 97,29 \\
\hline Fluxo Luminoso (lm) & 1906 & 1781 \\
\hline Potência (W) & 42,01 & 18,31 \\
\hline Fator de Potência & 0,87 & 0,99 \\
\hline THD & $10,6 \%$ & $3,13 \%$ \\
\hline
\end{tabular}

\subsection{Reprodução de cores}

A temperatura de cor das duas luminárias se enquadra na faixa de $5000 \mathrm{~K}$, mesmo a luminária LED apresentando uma temperatura de cor um pouco superior. Com relação ao índice de reprodução de cores (IRC), a luminária LED se enquadra na faixa de boa reprodução de cores, superior a 80, enquanto a fluorescente se enquadra na faixa de reprodução de cores razoável, ente 60 e 80 (Costa, 2006), isso implica em uma melhor representação das cores em ambientes iluminados por LED. Apesar de em muitos casos a diferença no índice de reprodução de cores não ser facilmente identificado, para alguns ambientes voltados para tarefas específicas, o desempenho com iluminação artificial com um IRC mais elevado pode ser sensivelmente superior. O espectro de luz do LED é caracterizado pelo azul $(450 \mathrm{~nm})$, como mostrado na figura 1, que é a estrutura básica do LED branco. A outra parte do espectro, representada na figura 1, é formada com a adição de fósforo no LED azul, já o espectro da lâmpada fluorescente, como mostrado na figura 2, é descontínuo e a curva depende do material luminescente (Baleja et al., 2015). 


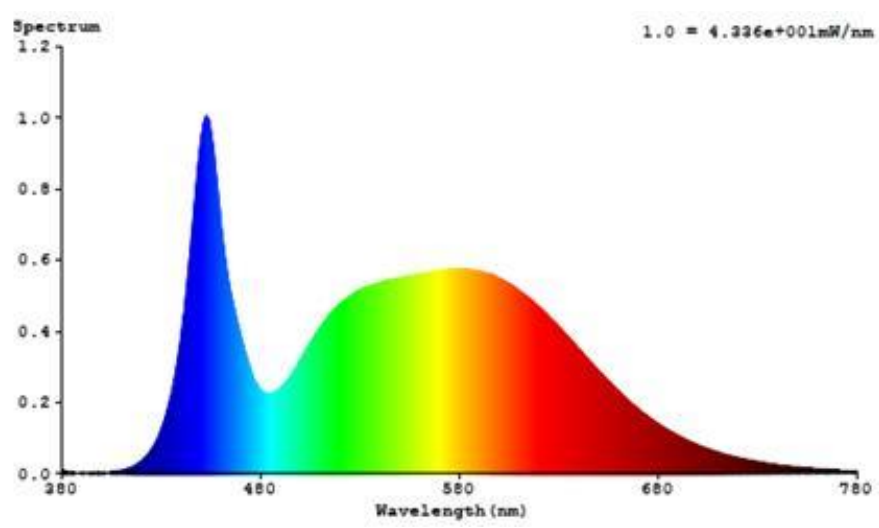

Figura 1 - Reprodução de Cor - Lâmpada LED

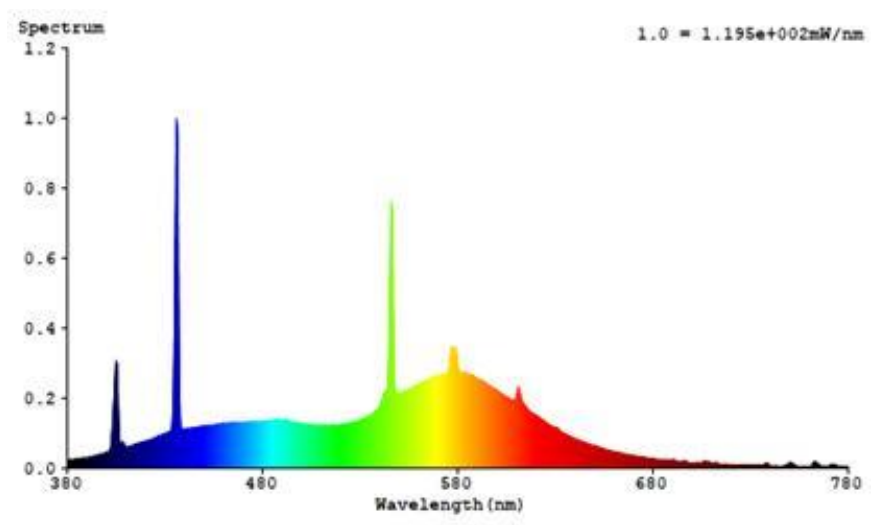

Figura 2 - Reprodução de Cor - Lâmpada Fluorescente

\subsection{Fator de Potência e THD}

Apesar de um bom desempenho da luminária fluorescente em relação ao fator de potência, o valor ainda é muito aquém se comparado às medições realizadas com a luminária LED. Quanto mais próximo do valor unitário estiver o fator de potência da embarcação, o gerador do navio irá operar em um ponto de maior eficiência e por consequência diminuirá os níveis de emissão de poluentes (Su e Liao, 2013). A produção de harmônicos dos equipamentos conectados na rede elétrica da embarcação pode prejudicar sobremaneira a qualidade da energia elétrica, de forma especial por se tratar de um sistema isolado devido ao valor reduzido da potência de curto circuito da instalação. Nesse sentido, o melhor desempenho da luminária LED é mais uma vantagem significativa da sua aplicação, pois as utilizações de reatores eletrônicos nas lâmpadas fluorescentes produzem um valor significativo de harmônicos de corrente, impactando negativamente na qualidade da energia elétrica de bordo, sendo um dos desafios enfrentados nos sistemas elétricos das embarcações (Skjong et al., 2016).

\subsection{Distribuição luminosa das luminárias}

Devido à semelhança das luminárias utilizadas para as duas tecnologias de lâmpada, as curvas polares de distribuição luminosa das figuras 3 e 4 , mostradas para os ângulos $0^{\circ}, 90^{\circ}, 180^{\circ}$ 
ISSN: $1415-7314$

ISSN online: $2317-6717$

e $270^{\circ}$, são bastante semelhantes. As luminárias são do tipo direta, ou seja, o fluxo luminoso é dirigido diretamente sobre a superfície a ser iluminada. A intensidade luminosa verificada na luminária fluorescente é um pouco superior, atingindo 439,6 cd comparada com os 408,9 cd da LED. O fluxo luminoso emitido pela luminária fluorescente também é um pouco superior ao da luminária LED, conforme os valores apresentados na tabela 1.

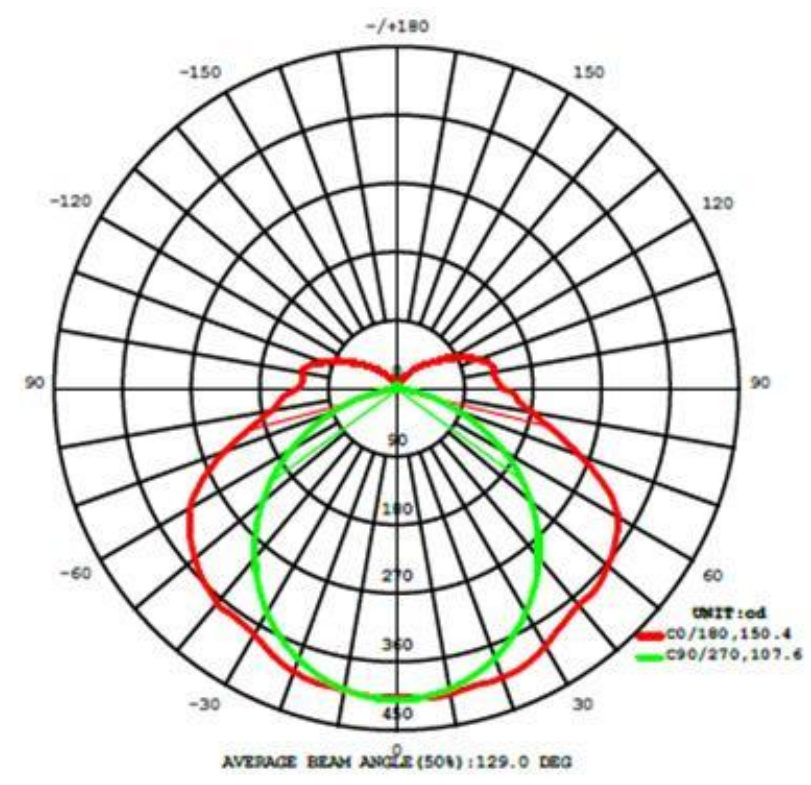

Figura 3 - Curva Polar de Distribuição Luminosa - Lâmpadas de LED

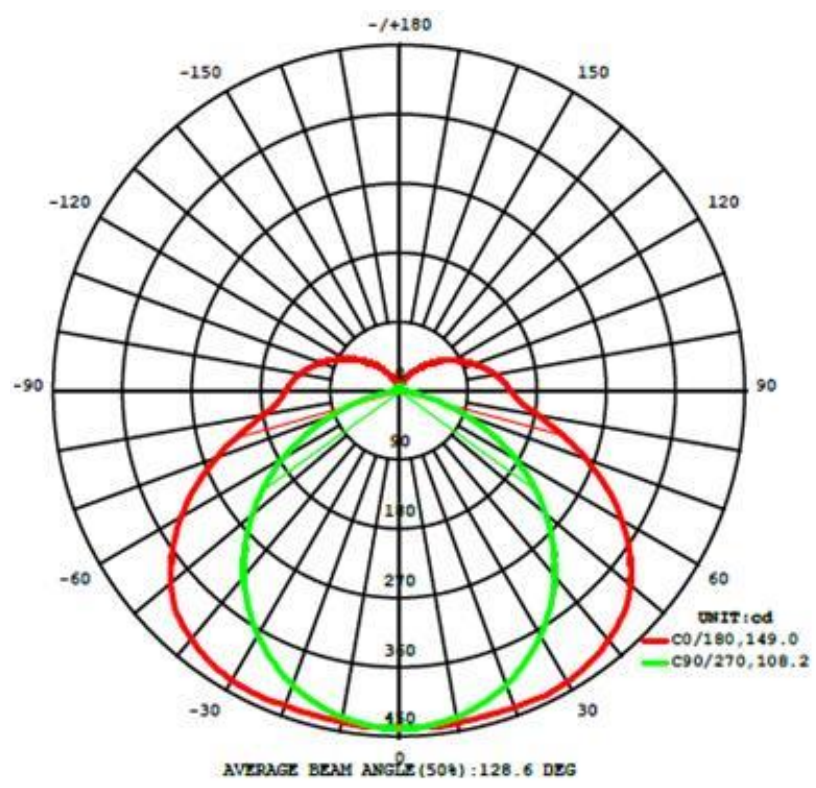

Figura 4 - Curva Polar de Distribuição Luminosa - Lâmpadas Fluorescentes

\subsection{Níveis de iluminância com utilização do LED}

A figura 5 mostra os níveis de iluminância nos diversos compartimentos do navio conseguidos com a utilização das lâmpadas LEDs. As simulações foram realizadas com as características das luminárias obtidas a partir dos testes realizados no Lablux. 

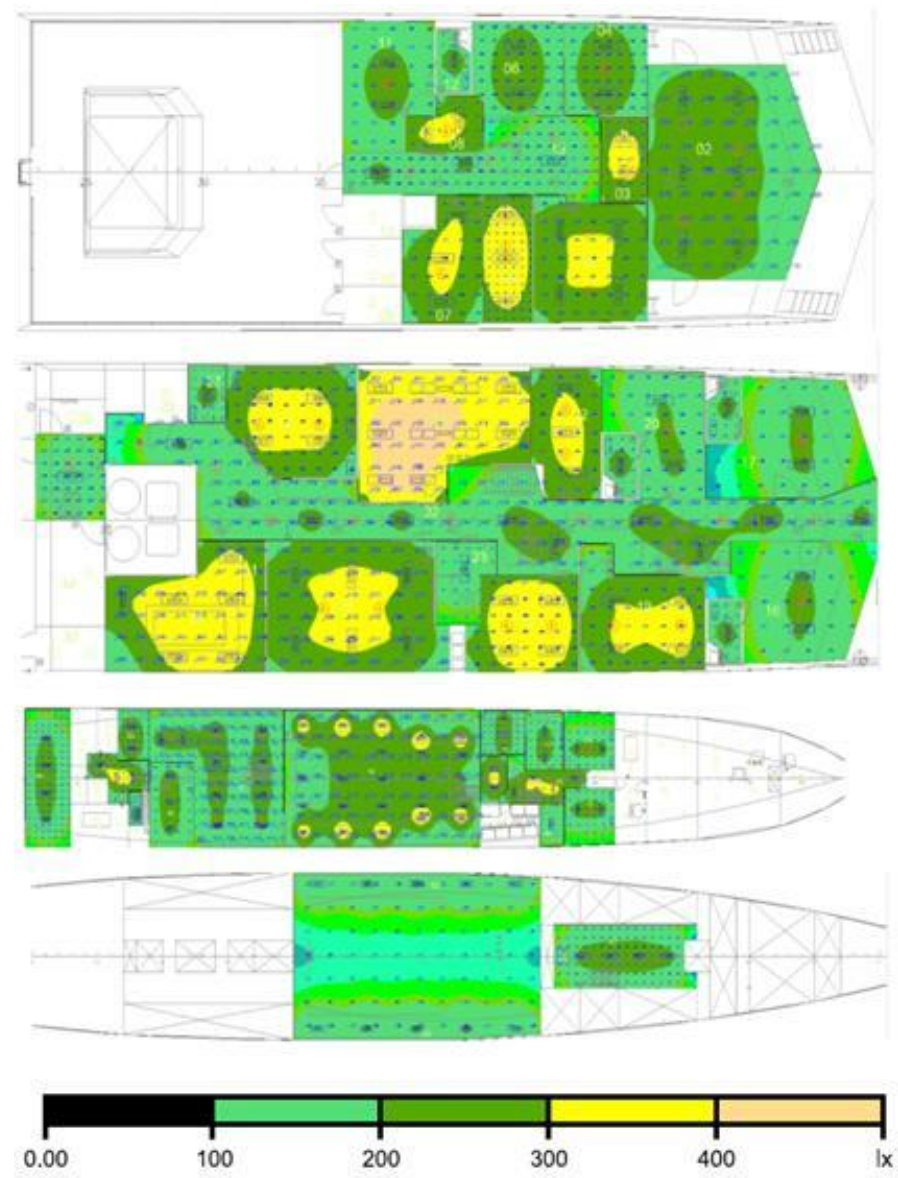

Figura 5 - Iluminância dos compartimentos do Navio com LED

\subsection{Eficiência Energética}

A principal justificativa para o investimento na substituição das lâmpadas presentes na embarcação é a eficiência energética proporcionada pela tecnologia proposta. A utilização de luminárias LED no projeto irá reduzir em aproximadamente $3,8 \mathrm{~kW}$ na potência instalada do navio. A quantidade de luminárias utilizadas no navio, mostrada na tabela 2 , foi obtida a partir dos dados de projeto, onde é verificado a iluminância mínima para navios de guerra. A economia representa $1 \%$ da potência nominal do gerador do navio. Os valores conseguidos com a economia são consideráveis, uma vez que a carga total de iluminação instalada na embarcação foi reduzida para menos da metade. Além do ganho direto de eficiência energética, há ainda diversas vantagens a ela associados, por exemplo, a diminuição de emissões da máquina térmica que aciona o gerador, ocorrido pela economia de combustível. Outro benefício inerente ao LED é o seu maior tempo de vida útil, reduzindo assim a necessidade do descarte em um intervalo de tempo reduzido, diminuindo necessidade de manutenção e aumentando o espaço nos compartimentos destinado a sobressalentes. 
Tabela 2 - Comparação das Luminárias Fluorescentes e LED

\begin{tabular}{|c|c|c|}
\hline & $\begin{array}{c}\text { Fluorescente } \\
(2 \times 20 \mathrm{~W})\end{array}$ & $\begin{array}{c}\text { LED } \\
(2 \times 10 \mathrm{~W})\end{array}$ \\
\hline $\begin{array}{c}\text { Potência da Luminária } \\
\text { (W) }\end{array}$ & 42,01 & 18,31 \\
\hline $\begin{array}{c}\text { Quantidade de } \\
\text { Luminárias }\end{array}$ & \multicolumn{2}{|c|}{159} \\
\hline Potência Total (W) & 6679,59 & 2911,29 \\
\hline $\begin{array}{c}\text { Potência Aparente } \\
\text { Total (VA) }\end{array}$ & 7767,69 & 2934,77 \\
\hline
\end{tabular}

\subsection{Análise econômica da substituição das tecnologias}

A tabela 3 apresenta um resumo do aspecto econômico na mudança da tecnologia das luminárias do navio. Apesar do maior investimento inicial para a adoção do LED, o custo irá se pagar rapidamente. A partir do perfil de operação da embarcação, foi estimado o custo de operação em um ano para cada tipo de lâmpada, de acordo com os resultados mostrados na tabela 3. Dessa forma haveria retorno do investimento com a implantação do LED já no primeiro ano. Associando a redução no consumo de combustível ao tempo de vida útil das tecnologias comparadas, a redução nos custos se torna ainda mais significativa. Em média, a cada 1,5 anos deverão ser trocadas as lâmpadas fluorescentes do navio, já para o LED o tempo médio entre trocas é maior, sendo de aproximadamente 3,5 anos. Considerando o tempo de vida útil de 30 anos do navio, a economia com a utilização do LED se dará tanto no gasto com combustível quanto na compra de sobressalentes, sendo todo o custo do sistema de LED cerca de 50,46\% do equivalente sistema fluorescente, o que representa uma economia de $\mathrm{R} \$ 464.500,00$ durante o tempo de vida útil do navio. É importante ressaltar que os valores apresentados fazem referência aos valores praticados atualmente, com o objetivo de obter uma ordem de grandeza da economia na operação da embarcação com a utilização do LED. Os resultados por hora apresentados dizem respeito a apenas um navio da classe. Caso a substituição seja implementada para todos os navios da classe, a economia conseguida, levando em consideração o tempo previsto para o navio operar, será substancial. 
Tabela 3 - Análise Econômica da Substituição das Lâmpadas Fluorescentes e LED

\begin{tabular}{|c|c|c|}
\hline $\begin{array}{c}\text { Custo Inicial para o } \\
\text { navio (R\$) }\end{array}$ & $\begin{array}{c}\text { Fluorescente } \\
(2 \times 20 \mathrm{~W})\end{array}$ & $\begin{array}{c}\text { LED } \\
(2 \mathrm{x} 10 \mathrm{~W})\end{array}$ \\
\hline $\begin{array}{c}\text { Tempo de Vida Útil } \\
\text { (h) }\end{array}$ & $1.003,76$ & $21.862,52$ \\
\hline $\begin{array}{c}\text { Energia de Consumida } \\
\text { (kWh) - Ciclo de } \\
\text { Operação Anual }\end{array}$ & $30.294,39$ & $13.203,77$ \\
\hline $\begin{array}{c}\text { Custo da Energia (R) } \\
\text { - }\end{array}$ & $21.870,82$ & $9.532,37$ \\
$\begin{array}{c}\text { Ciclo de Operação } \\
\text { Anual }\end{array}$ & & $504.596,30$ \\
\hline $\begin{array}{c}\text { Custo da Energia e } \\
\text { Lâmpadas }\end{array}$ & $937.999,80$ \\
$\begin{array}{c}\text { Sobressalentes (R\$) - } \\
\text { Ciclo de Operação do } \\
\text { Navio }\end{array}$ & & \\
\hline
\end{tabular}

\section{Conclusão}

Este artigo apresentou as implicações, para um navio patrulha de pequeno porte, da substituição de lâmpadas fluorescentes por lâmpadas LEDs. Os benefícios inerentes à utilização das lâmpadas LEDs ficaram evidenciados a partir dos testes realizados com as duas tecnologias comparadas. Destaca-se, que para rede elétrica de bordo, houve uma melhora na qualidade de energia elétrica, com uma redução de aproximadamente $70 \%$ da distorção harmônica, e fator de potência, que teve um aumento de $17 \%$. Com isso verificou-se que o desempenho das lâmpadas de LED foi muito superior às lâmpadas fluorescente, pois além de melhorar a eficiência energética da embarcação estudada, implicando assim na redução de consumo e emissões de poluentes, também se pode observar que o tempo de vida é muito superior, diminuindo assim tempo de manutenção, para a troca das mesmas.

A partir do perfil de operação e tempo de vida útil do navio, calculou-se uma redução de $50 \%$ do custo total de geração e sobressalentes, verificando assim a viabilidade econômica da substituição, gerando economia na operação do navio.

Sendo assim, para o navio em que o estudo de caso se baseou, a troca do sistema de iluminação trará vários benefícios caso sua implementação seja realizada.

\section{Referências}

BALEJA, R.; SUMPICH, J.; BOS, P.; HELSTYNOVA, B.; SOKANSKY, K; NOVAK, T. Comparison of LED Properties, Compact Fluorescent Bulbs and Bulbs in Residential Areas, In: $201516^{\text {th }}$ International Scientific Conference on Electric Power Engineering (EPE), 2015. doi: 10.1109/EPE.2015.7161181. 
BOVERI, A.; SILVESTRO, F.; GUALENI, P. Ship Electrical Load Analysis and Power Generation Optimisation to Reduce Operational Costs. In: International Conference on Electrical Systems for Aircraft, Railway, Ship Propulsion and Road Vehicles \& International Transportation Electrification Conference (ESARS-ITEC), 2017. doi: 10.1109/ESARSITEC.2016.7841422.

BRAVO, R. J.; ABED, N. Y. Experimental Evaluation of the Harmonic Behavior of LED Light Bulb, In: IEEE Power \& Energy Society General Meeting (PES), 2013. doi: 10.1109/PESMG.2013.6672991.

BROOKS, M. S.; TRIBBLE, A. L. Stakeholder Values and Perspectives when Implementing LED Lights on Navy Ships, Master of Business Administration from Naval Postgraduate School, 2014. Disponivel em: http://www.dtic.mil/dtic/tr/fulltext/u2/a608008.pdf.

CIZEC, C. J. Shipboard LED lighting: A business case analysis, Master of Business Administration from Naval Postgraduate School Monterey, 2009. Disponivel em: http://www.dtic.mil/dtic/tr/fulltext/u2/a514251.pdf.

COSTA, J.C. Iluminação Econômica, $4^{\circ}$ ed, EDIPUCRS, Porto Alegre 2006.

DEW, N.; ATEN, K.; FERRER, G. How many admirals does it take to change a light bulb? Organizational innovation, energy efficiency, and the United States Navy's battle over LED lighting, Energy Research \& Social Science, v.27, 57-67, 2017. doi: 101016/j.erss.2017.02.009.

FREYMILLER, A. T. LED Shipboard Lighting: A Comparative, Master of Business Administration from Naval Postgraduate School Monterey, 2009. Disponivel em: www.dtic.mil/cgi-bin/GetTRDoc?AD=ADA514268.

INMETRO. Portaria n. 489, de 8 de dezembro de 2010, 2010.

INMETRO. Portaria n. 389, de 25 de agosto de 2014, 2014.

INMETRO. Portaria n. 143, de 13 de março de 2015, 2015.

MARPOL. MARPOL 73/78 - ANEXO 1, Regras para a Prevenção da Poluição por Óleo. 2018. Disponivel em: https://www.ccaimo.mar.mil.br/sites/default/files/marpol_anexo1-11ago.pdf.

McCOY, M. NAVSEA Reducing Fleet Energy Consumption. Currents, p.58-62, 2012.

MORTON,J.F.; TRUVER,S.C. The Great Green Fleet Naval Warfighting Imperatives for Energy Security. Defense AT\&L, 2011.

SARRIS, E. Naval Ship Propulsion and Electric Power Systems Selection for Optimal Fuel Consumption, Master of Science in Engineering and Management ARCHNES at the Massachusetts Institute of Technology, 2011.

SKJONG, E.; VOLDEN, R.; RODSKAR, E.; MOLINAS, M. Past, Present, and Future Challenges of the Marine Vessel's Electrical Power System, IEEE Transactions on Transportation Electrification, v.2, p.1-16, 2016.

SKJONG, E.; JOHANSEN, T. A.; MOLINAS, M.; SORENSEN. A. J. Approaches to Economic Energy Management in Diesel-Electric Marine Vessels, IEEE Transactions on Transportation Electrification, v.3, p.22-35, 2017. doi: 10.1109/TTE.2017.2648178.

SOLÀ, J. C.; SALICHS, M. Study and Characterization of Waveforms from Low-Watt $(<25 \mathrm{~W})$ Compact Fluorescent Lamps with Electronic Ballasts, IEEE Transactions on Power Delivery, v.22, p.2305-2311, 2007. doi: 10.1109/TPWRD.2007.899551. 
ISSN: $1415-7314$

ISSN online: $2317-6717$

SU, C. L.; LIAO, M.C. A Method for Evaluating Energy Efficiency to Justify Power Factor Correction in Ship Power Systems, IEEE Transactions on Industry Applications, v. 49, p.27732782, 2013. doi: 10.1109/TIA.2013.2265293. 